\title{
Young children, but not chimpanzees, are averse to disadvantageous and advantageous inequities
}

\author{
Julia Ulber ${ }^{\mathrm{a}, *}$, Katharina Hamann ${ }^{\mathrm{a}}$, Michael Tomasello ${ }^{\mathrm{a}, \mathrm{b}}$ \\ ${ }^{a}$ Department of Developmental and Comparative Psychology, Max Planck Institute for Evolutionary Anthropology, 04103 \\ Leipzig, Germany \\ ${ }^{\mathrm{b}}$ Department of Psychology and Neuroscience, Duke University, Durham, NC 27708, USA
}

\section{A R T I C L E I N F O}

\section{Article history:}

Received 29 August 2016

Revised 31 October 2016

Available online 2 December 2016

\section{Keywords:}

Inequity aversion

Fairness

Social context

Resource allocation

Pan troglodytes

Cooperation

\section{A B S T R A C T}

The age at which young children show an aversion to inequitable resource distributions, especially those favoring themselves, is unclear. It is also unclear whether great apes, as humans' nearest evolutionary relatives, have an aversion to inequitable resource distributions at all. Using a common methodology across species and child ages, the current two studies found that 3- and 4-yearold children $(N=64)$ not only objected when they received less than a collaborative partner but also sacrificed to equalize when they received more. They did neither of these things in a nonsocial situation, demonstrating the fundamental role of social comparison. In contrast, chimpanzees $(N=9)$ showed no aversion to inequitable distributions, only a concern for maximizing their own resources, with no differences between social and nonsocial conditions. These results underscore the unique importance for humans, even early in ontogeny, for treating others fairly, presumably as a way of becoming a cooperative member of one's cultural group.

(c) 2016 Elsevier Inc. All rights reserved.

* Corresponding author.

E-mail address: ulber@eva.mpg.de (J. Ulber). 


\section{Introduction}

It is a striking fact that humans consider a given amount of resources perfectly satisfactory unless someone else gets more, in which case they often find that amount unsatisfactory. It is an even more striking fact that humans consider a given amount of resources perfectly satisfactory unless someone else gets less, in which case again they often find that amount unsatisfactory. In the language of economics, humans are averse to inequity-in both advantageous and disadvantageous directions (e.g., Dawes, Fowler, Johnson, McElreath, \& Smirnov, 2007; Fehr \& Schmidt, 1999).

When and how young children become averse to inequitable resource distributions, especially those favoring themselves, is a matter of some controversy. Various studies report different results depending on the context in which children are observed, the role of adults in the process, and the alternatives children have at their disposal in the test situation. Most straightforward, in thirdparty situations where they must distribute resources among others, children from as young as 3 years show a very strong bias for equal distributions (e.g., Frydman \& Bryant, 1988; Olson \& Spelke, 2008; Peterson, Peterson, \& McDonald, 1975; Rochat et al., 2009; Shaw \& Olson, 2012). When young children themselves are among the recipients, however, the situation becomes more complicated.

With regard to distributions that disadvantage them, young children show negative responses from as young as 3 years (Birch \& Billman, 1986; LoBue, Nishida, Chiong, DeLoache, \& Haidt, 2011). By 4 or 5 years of age, children will even sacrifice own rewards or accept an absolutely lesser offer to avoid being at a disadvantage relative to a peer (Blake \& McAuliffe, 2011; Sheskin, Bloom, \& Wynn, 2014). It is important that a number of measures in some of these studies contradict the hypothesis that children just want more resources. Rather, they are making an explicit social comparison between themselves and others and are invoking words like unfair if they are disadvantaged. However, McAuliffe, Blake, Kim, Wrangham, and Warneken (2013) found that children in this age range also rejected disadvantageous distributions when there was no peer partner present (i.e., an adult put less resources on the child's tray but put more on an opposite tray that belonged to nobody), suggesting that at least some of children's judgments of unfairness may be directed at the adult doing the distributing.

In the case of advantageous distributions, Blake and McAuliffe (2011; see also Blake, McAuliffe, \& Warneken, 2014; Blake et al., 2015; McAuliffe et al., 2013) used an apparatus in which children could either accept an allocation between themselves and a peer or else reject it so that no one got anything. They found that children did not reject advantageous inequities until 8 or 9 years of age. In that study, children were not allowed to respond by suggesting a redistribution because, the authors argued, a redistribution would potentially confound the motive of sympathy for the partner with aversion to inequity (see Box 2 in Blake et al., 2014). But this method has its own confound in that rejecting an inequitable distribution punishes both partners (because acceptance of the distribution benefits both to some degree) and also wastes resources. This raises the possibility that young children might dislike inequity, just not enough to punish everyone. And in fact, when children are allowed to reallocate advantageously distributed resources, from as young as 3 years they sometimes sacrifice their extra resources to equalize. Specifically, in the second study of Hamann, Warneken, Greenberg, and Tomasello (2011; see also Ulber, Hamann, \& Tomasello, 2015; Warneken, Lohse, Melis, \& Tomasello, 2011), when a pair of 3-year-olds collaborated to produce resources and one child unexpectedly received more than the other, the lucky child sacrificed to equalize nearly $80 \%$ of the time. Moreover, in a control condition where the two children produced resources independently (the study was focused on the effect of collaboration), the lucky child still sacrificed to equalize nearly $40 \%$ of the time. Notably, in this study no adult was present at the time of distribution, and no adult gave the children instructions about how to distribute the resources.

In seeking explanations for children's proclivity for equal resource distributions, a crucial source of information is the behavior of humans' closest living relatives, the great apes. If they show a similar proclivity, it becomes highly unlikely that children's tendencies result solely from adult socialization or teaching; if they show no such proclivity, humans' aversion to inequity would seem to be evolutionarily unique, with developmental mechanisms still to be determined. So far, evidence is mixed. Although chimpanzees engage in some food sharing in the wild, (Boesch, 1994; Mitani \& Watts, 2001), it is not clear that they are doing more than simply avoiding harassment from beggars (Gilby, 2006). In 
experimental research, Brosnan and colleagues reported that chimpanzees reject unequal resource distributions between themselves and others (Brosnan, Schiff, \& de Waal, 2005; Brosnan, Talbot, Ahlgren, Lambeth, \& Schapiro, 2010), but Bräuer and colleagues found that what was driving the chimpanzees' rejections was not unequal distributions but rather the fact that the subject was getting unpalatable food when better food was apparently available (i.e., the effect was driven not by social comparisons but by food comparisons) ${ }^{1}$ (Bräuer, Call, \& Tomasello, 2006, 2009; see also Hopper, Lambeth, Schapiro, \& Brosnan, 2013). In an ultimatum game paradigm, Jensen, Call, and Tomasello (2007) and Kaiser, Jensen, Call, and Tomasello (2012) reported that both chimpanzees and bonobos accepted any food offer no matter how poor their share was in comparison to their partner's share. In a food sharing paradigm, chimpanzees seemed to focus exclusively on the food that they themselves might get, not caring about what others were receiving (Jensen, Hare, Call, \& Tomasello, 2006; Silk et al., 2005). And in Hamann and colleagues' (2011) collaboration study, chimpanzees competed over the spoils of a collaborative effort, rather than sharing them, in repeated attempts to monopolize the rewards for themselves.

Overall, then, the findings with children show some discrepancies between experimental paradigms, especially with regard to their reactions to advantageous inequities, with the studies of Blake and colleagues suggesting that aversion to advantageous inequity emerges only at 8 years of age but the studies of Hamann, Warneken, and colleagues suggesting that in collaborative situations this aversion appears at 3 years of age. And the findings with great apes also show different findings with different paradigms, although the preponderance of evidence is certainly that they are not averse to inequity per se. However, in nearly all of the ape paradigms, subjects needed to overcome their own selfish desire for good food in order to be equitable with a conspecific partner-and so a response measure minimizing this competing motivation would be desirable.

Given these various results from different experimental paradigms, in the current study we wanted to look systematically at both young children's and chimpanzees' reactions to both advantageous and disadvantageous resource distributions in a task situation with minimal competing demands. In our task, children or chimpanzees either collaborated with a partner to produce the different distributions or not, and then after collaboration they could either reel in the resulting distribution or not. After non-collaboration (the distribution was simply made available), again children or chimpanzees could either reel in the distribution or reject it, but in this case there was no partner on the other side-so no social comparison (and hence no sense of social inequity) was possible. Because rejection might require advanced inhibitory or impulse control skills (especially difficult for young children and chimpanzees; e.g. Steinbeis, Bernhardt, \& Singer, 2012), the reeling in response also allowed us to look at children's or chimpanzees' reluctance or conflict to accept a distribution, operationalized as the speed at which they reeled in the distribution. Moreover, we also looked at their attempts to reallocate the distribution, matching the general definition of inequity aversion as the tendency to dislike and correct unequal outcomes (Fehr \& Schmidt, 1999).

We expected children to show inequity aversion toward both advantageous and disadvantageous payoffs when in a social situation but to show no systematic refusal of any offers under nonsocial circumstances. We expected chimpanzees to show no such differentiation. We also expected children's inequity aversion-especially of the advantageous variety-to be more marked at 3 or 4 years of age than at 2 years of age because older children are better able to overcome their competing selfish tendencies.

\section{Study 1}

\section{Method}

\section{Participants}

Participants were 32 3.5-year-old children (range $=3 ; 04-3 ; 11$ [years; months], $M=3 ; 09,16$ girls) and 32 4.5-year-old children (range $=4 ; 04-4 ; 11, M=4 ; 08,16$ girls). One additional 3-year-old child

\footnotetext{
${ }^{1}$ Brosnan and de Waal (2003) also reported positive results in a similar paradigm with capuchin monkeys, but several other laboratories again reported that the behavior is driven not by social comparison but rather by food comparison: Dubreuil, Gentile, and Visalberghi (2006), Fontenot, Watson, Roberts, and Miller (2007), McAuliffe and colleagues (2015), Roma, Silberberg, Ruggiero, and Suomi (2006), and Sheskin, Ashayeri, Skerry, and Santos (2014).
} 
was excluded from analysis due to experimenter error. Two 4-year-olds needed to be excluded due to apparatus or camera malfunction. All children were native German speakers, came from mixed socioeconomic backgrounds, and were recruited from a child database of a medium-sized German city. All testing was done in a laboratory room. Written parental consent was given for all participants.

\section{Design}

Children were randomly assigned to either the social or nonsocial condition, counterbalanced for gender and age group. In both conditions, children were presented with four different resource allocations in a $2 \times 2$ design (see Table 1). Each allocation was presented in a block of two trials, resulting in eight trials in total. Block order was counterbalanced, with equal and unequal payoffs alternating.

\section{Apparatus}

Children played a game in which they could accept or reject various reward allocations. To retrieve the rewards, children needed to operate an apparatus (see Fig. 1 for details). The upper half of the apparatus consisted of a board. Rewards were placed in the center of that board and needed to be shoved toward the end, where they fell down and ended up in two cups. Shoving was done either collaboratively by the child and a puppet (social condition) or by the experimenter (nonsocial condition). The experimenter could manipulate the number of rewards that fell into each cup without children's awareness. For instance, six rewards on the flat could "accidently" result in a 5-1 split (leaving five rewards in children's cup and one reward in the other cup) or a 1-5 split. Children could then decide whether to accept the allocation by turning a crank in one direction such that the rewards landed in outside bowls. Alternatively, they could reject the outcome by cranking in the opposite direction. In that case, rewards disappeared. Chocolate candies (Smarties) were used as rewards throughout the study.

\section{Procedure}

During a short warm-up period, children engaged in free play with the experimenter to get familiar with each other. If children were assigned to the social condition, they were joined by a puppet (a 25$\mathrm{cm}$ bee hand doll: "Bella" for girls or "Eddie" for boys) operated live by a second experimenter. The reason for introducing a puppet and not a peer was to control and standardize the reactions of the social partner for any behavior of children. Using hand puppets in studies where peer partners are not feasible is a common procedure when studying young children (see Gräfenhain, Carpenter, \& Tomasello, 2013; Rakoczy, Warneken, \& Tomasello, 2009; Ulber, Hamann, \& Tomasello, 2016) and has the advantage that children of that age, unlike adults, do not perceive the puppet as a natural authority but rather treat the puppet as a peer, ignoring the adult puppeteer.

When children felt comfortable with their environment and explored the warm-up room freely without their parents, testing began in the study room. One caregiver was present during the testing but was instructed not to interfere with the procedure. Instead, the caregiver was asked to pretend to read a magazine.

Children were placed on the right side of the apparatus. The puppet (if part of the game) was placed on the left side. Testing consisted of a demonstration phase and a test phase and was conducted over several trials. After every trial, parents were asked to distract and play with their children so that the experimenter could bait the apparatus again in order to (a) keep children unaware of the experimenter's manipulation of the apparatus and (b) prevent children and the puppet from playing together in between trials to control for their amount of shared experiences and familiarity in contrast to the nonsocial condition. That is, in the social condition children and the puppet played parallel to but not with each other during the breaks, whereas in the nonsocial condition children played alone (with their parents). Each session was videotaped and lasted a maximum of $30 \mathrm{~min}$.

Demonstration phase. In the social condition, the experimenter explained the apparatus to both children and the puppet together. Accordingly, the bowl on the left side was introduced as the puppet's, whereas the bowl on the right side was announced as children's. In the nonsocial condition, the experimenter explained the apparatus exclusively to children because no play partner was present. This time the bowl on the left side had a lid, such that whatever fell into it got lost and was not accessible 
Table 1

Four possible resource allocations.

\begin{tabular}{lll}
\hline & Equal & Unequal \\
\hline Child gets 5 & $5-5$ & $5-1$ \\
& High value equality & Advantageous inequity \\
Child gets 1 & $1-1$ & $1-5$ \\
& Low value equality & Disadvantageous inequity \\
\hline
\end{tabular}

anymore (i.e., like a trash bin). During the demonstration phase, children first learned to operate the crank toward the two directions. For demonstration purposes, the cups were baited with colored dice or paper pellets. Children were encouraged to "accept" the dice but were asked to "reject" the paper pellets. Each direction was trained twice (Trials 1-4).

In a next step, the experimenter gave two Smarties to children and, if present, two Smarties to the puppet to be eaten right away or collected in a paper bag (Trial 5). Subsequently, more Smarties were placed on the upper board and needed to be shoved forward (either by children and the puppet together or by the experimenter, depending on the condition). Eventually, two Smarties fell into each cup. In Trial 6 children were encouraged to get the Smarties out, whereas in the final demonstration trial children were asked to make them disappear "for fun." The latter was especially important to make children feel comfortable with "discarding" food and to show children that they would not face any negative consequences if they decided to reject the candies. Nearly all children proceeded to the test phase subsequently to the last training trial. Only three dyads needed either one additional training trial ( $n=2$; additional training with cranking directions) or two additional training trials $(n=1$; additional encouragement to crank due to shyness) to enter the test phase.

Test phase. The test phase applied four different allocations of Smarties (see Table 1) over eight trials. Each trial began with the experimenter pointing out the new Smarties that were placed in the center of the board. In a next step, the Smarties were shoved down. As soon as the Smarties landed in the two cups, the experimenter asked children whether they got many or few candies and whether the puppet got many or few candies (social condition) as well as whether the other cup contained many or few candies (nonsocial condition). Children were not questioned if they correctly stated the number of Smarties in each cup spontaneously. If children gave the wrong answer, the experimenter helped them count the Smarties and repeated her questions. Next, the experimenter released the crank: "And now it's your turn to decide: Do you want to get the Smarties out or make them disappear? Go ahead!" The experimenter turned her gaze away from children until the end of the trial.

If children accepted the allocation in the social condition, the puppet pointed out that she received either many or just a few Smarties and collected them one by one in her paper bag. In the nonsocial condition, the experimenter commentated that either many or few Smarties just landed in the trash bowl but that they were gone. These statements were done in a neutral descriptive tone, carefully avoiding any sort of prompting, in order to draw children's attention to the bait in the opposite cup. Pilot data revealed that children were often rather distracted and too excited by receiving candies and forgot to pay attention to what was happening on the other side, although they had correctly stated the number of Smarties in each cup before. If children rejected the allocation, the experimenter said that the candies had now disappeared and that, accordingly, nobody would get anything.

We also tried to provide a rather neutral yet natural reaction to any other behavior of children. If children requested a candy from the puppet, the puppet said that she would rather keep her Smarties and continued collecting them in her bag. If children requested more rewards from the experimenter, the experimenter said that she had no candies left for this round but that she would look to see whether there were any Smarties left to play with for a new trial. In cases where children snuck away Smarties from either the puppet or the bowl or tried to do so, after $5 \mathrm{~s}$ either the puppet commented on it ("These are my Smarties!") or the experimenter commented on it ("These Smarties are in the trash; they don't belong to anybody") but did not prevent children from doing so. If children shared Smarties, the puppet silently took them and put them in her bag but did not thank or praise the children. This was done in order to ensure that participants' decisions and behavior were based on the 


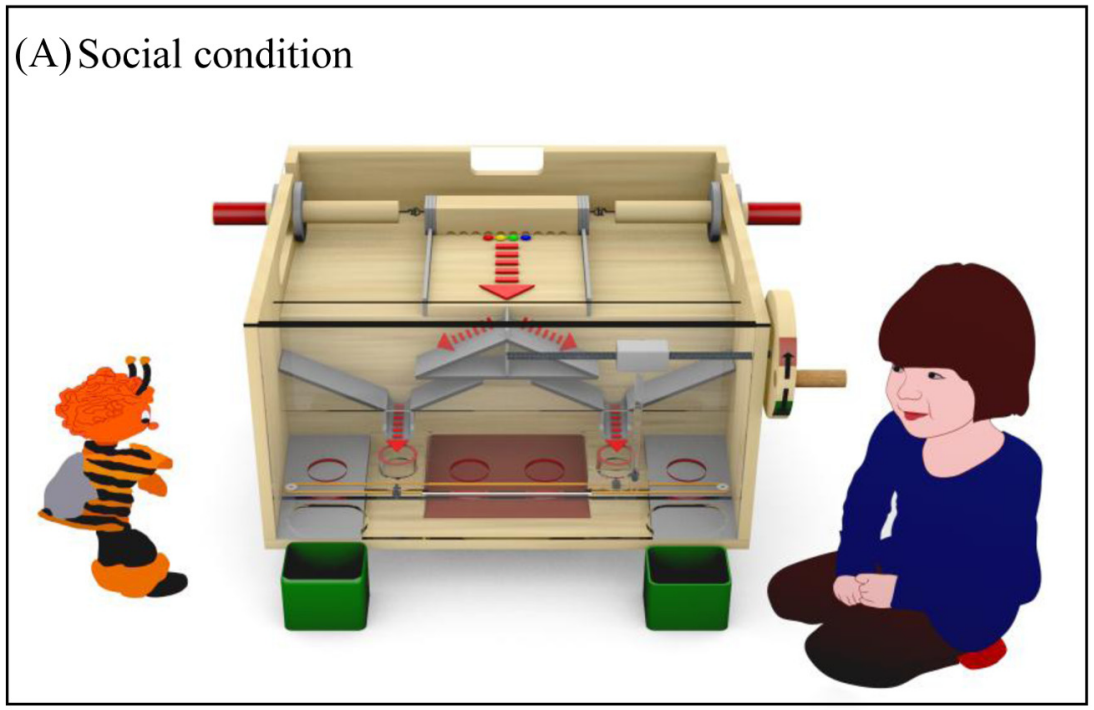

\section{(B) Nonsocial condition}

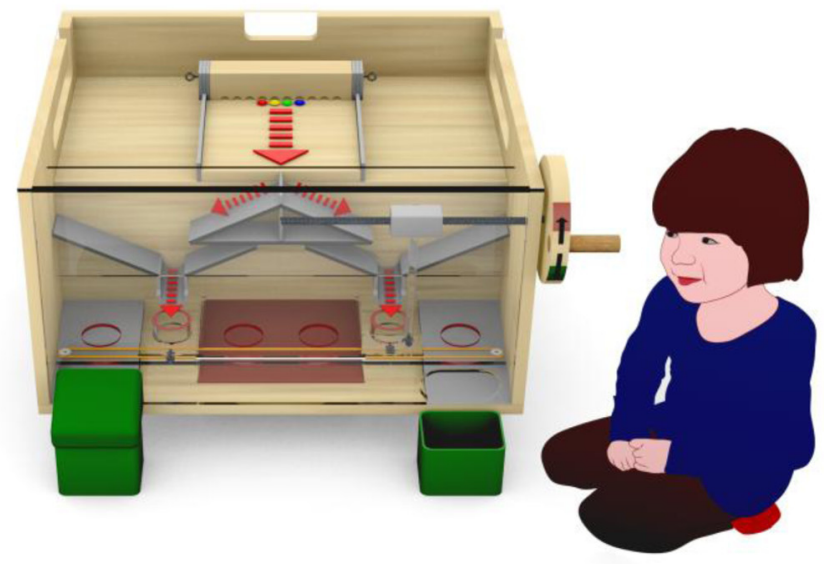

Fig. 1. Illustration of the test apparatus in the social condition (A) and nonsocial condition (B) in Studies 1 and 2. Children were placed on the right side of the apparatus, while the puppet (if present) sat on the left side of the apparatus. The experimenter sat behind the apparatus. Smarties were placed in the center of the board and could not be reached. A little platform was used to shove the Smarties forward and cause them to fall onto two plates. In the social condition, the platform had two handlebars attached on either side and could be moved forward only if both children and the puppet shoved their handlebar forward simultaneously. Otherwise, the board got stuck. Given the size of the apparatus, children were not able to touch and hence operate both handlebars alone. In the nonsocial condition, the experimenter operated the platform with a stick that pushed the board forward. With the help of a slider, the final split-up, and thus the final bait of the plates, could be manipulated without children's awareness. Children could operate the crank on their side of the apparatus. Cranking toward the green direction caused the plates to move outward, delivering Smarties to two outside bowls ("accepting an allocation"). Cranking toward the brown direction caused the plates to move inward, delivering Smarties inside the apparatus from where they were not accessible any longer ("rejecting an allocation"). Directions were marked with colors and arrows. (For interpretation of the references to color in this figure legend, the reader is referred to the Web version of this article.) 
resource distribution rather than being reinforced or influenced by the puppet. A trial ended when all rewards were either eaten or collected.

\section{Coding and data analysis}

Coding was done from video by the experimenter. One quarter (25\%) of the sample was randomly selected and coded for reliability by a second observer who was unaware of the study hypotheses. Primary coding focused on children's aversive behavior. Aversive behaviors consisted of the following behavioral categories: rejections, sharing Smarties after acceptance (i.e., by giving one or more Smarties to the puppet or placing them into the other bowl), and requesting more Smarties (including asking the puppet or experimenter for more Smarties and directly taking Smarties away, either by stealing them from the puppet or by sneaking out some candies that fell into the trash bowl). Attempted aversive actions were also coded as such if the intention was clear (e.g., children clearly reaching for one of the puppet's Smarties but being too slow to collect it before the puppet put it inside her bag). The three behaviors-reject, share, and request-were then summarized as aversive behavior and binary coded.

To provide a sensitive measure for their general motivation to receive the rewards, we also coded the cranking duration from the moment the experimenter released the crank and children started to turn it until the first candy fell into children's bowl. Furthermore, we analyzed children's verbal statements and coded whether children labeled the content of the cups correctly (e.g., few, many, five) and whether they used any language indicating a comparison between the two baits (e.g., more, less, too). The inter-rater agreement for aversive behavior was excellent $(\kappa=.95)$. The intraclass correlation coefficient for cranking duration was very good $(r=.97)$, indicating a strong consistency between the coders. Reliability for language coding was also very high $(\kappa=.95)$.

The main analysis was done using generalized linear mixed models (GLMMs; see Baayen, Davidson, \& Bates, 2008). All models were fitted in R using the package lme4 (Bates, Maechler, Bolker, \& Walker, 2014). Specifically, with aversive behavior as the binary response, we tested a model including condition (social or nonsocial), reward allocation (1-1,5-5, 1-5, or 5-1), age (3-year-olds or 4-year-olds), gender, and trial number $(1,2,3,4,5,6,7$, or 8$)$. Child identity was fitted as a random effect to control for repeated measures. We examined significance using both likelihood ratio tests (LRTs) by comparing the fit of the full model with that of the respective reduced model and the $p$-values provided by the final model. The general procedure was as follows. First, we created a full model that included all possible predictor variables and their interactions. Second, we compared the full model with a null model including only child identity and trial number to test whether the inclusion of predictors provided a better fit to the data overall. Unless noted otherwise, all full models provided a better fit to the data than null models. The tested models also did not differ significantly in goodness of fit from a model in which the effect of reward allocation was allowed to vary by individual and trial number (included as crossed random effects on the slope). Third, the final model was created from the full model by sequentially dropping single terms from the model and testing whether their inclusion improved the model fit. Children's cranking duration was analyzed in the same way by applying cranking time as the quantitative response variable.

\section{Results}

\section{Aversive behavior}

Results for aversive behavior are illustrated in Fig. 2. The final model revealed no effect of trial number, age group, or gender (all $p s>.29$ ). However, we found a significant interaction between condition and allocation, $\operatorname{LRT}(3)=17.45, p=.001$. Post hoc analysis, conducted separately for each condition, revealed that children in the social condition were more likely to react aversively if the offered allocation was unequal (either $5-1$ or $1-5$ ) compared with an equal allocation (5-5 or 1-1). In contrast, aversive behavior did not differ between allocations in the nonsocial condition (see Table 2). To rule out a possible underlying interaction between value and equality, we split up the previous factor reward allocation into the two factors: equality (equal or unequal) and value (high-child gets 5 or low-child gets 1). Mirroring the initial results, the final model showed a significant interaction 


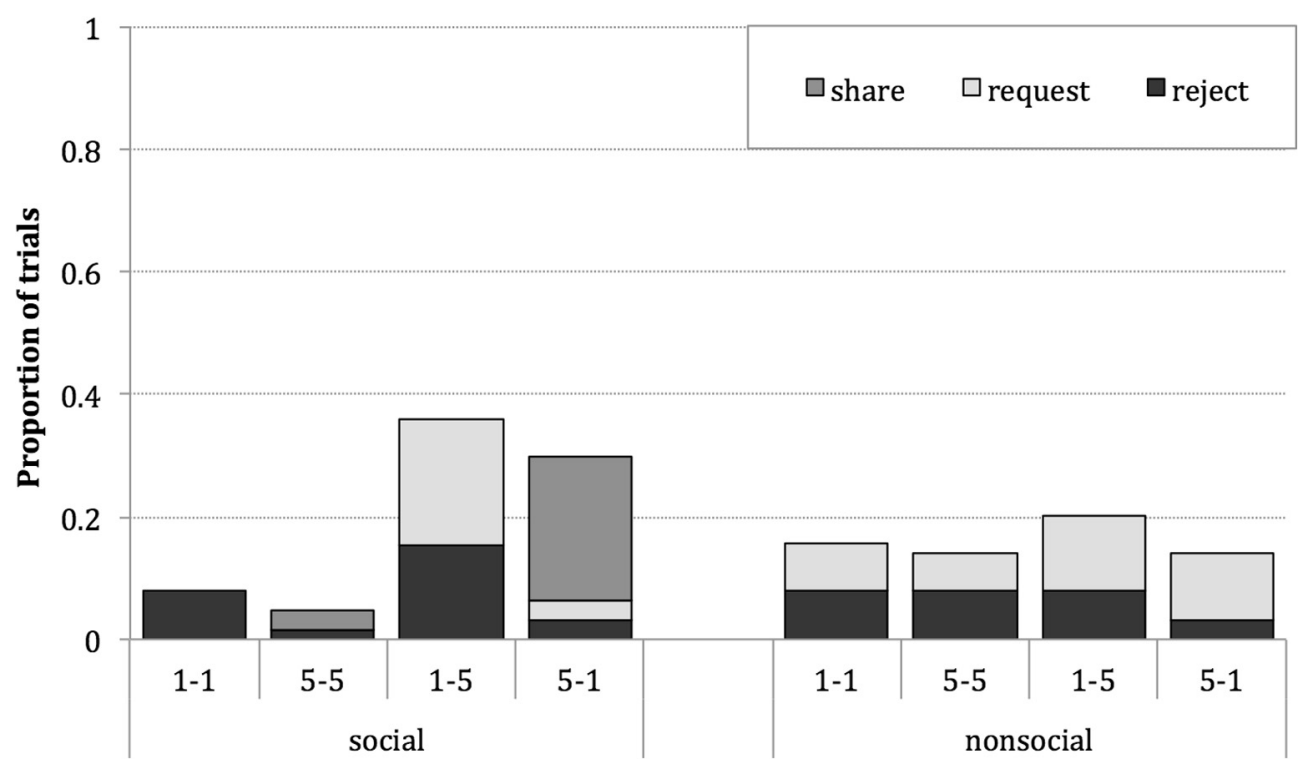

Fig. 2. Proportions of trials in Study 1 where children showed aversive behavior (sharing, requesting, or rejecting) toward four different resource allocations (1-1, 5-5, 1-5, and 5-1).

Table 2

Post hoc pairwise comparisons of aversive behavior across all allocations.

\begin{tabular}{lllll}
\hline & $1-1$ & $5-5$ & $1-5$ & $5-1$ \\
\hline $1-1$ & - & .376 & $<.001^{* * *}$ & $<.001^{* * *}$ \\
$5-5$ & .788 & - & $<.001^{* * *}$ & $<.001^{* * *}$ \\
$1-5$ & .435 & .295 & - & .293 \\
$5-1$ & .780 & .992 & .294 & - \\
\hline
\end{tabular}

Note. Results for the social condition are shown highlighted in grey above the diagonal, and results for the nonsocial condition are shown below the diagonal $\left({ }^{* * *} p<.001\right)$.

between equality and condition, $\operatorname{LRT}(1)=17.15, p<.001$, but no effect of or interaction with resource value $(p=.19)$.

Addressing only rejection rates, we found that children generally were more likely to reject an allocation when they received only one candy ( $1-1$ and $1-5 ; M=.10)$ than when they got five candies (5-5 and $5-1 ; M=.04$ ) irrespective of condition or age group (GLMM, main effect of resource value, estimate $=1.03, z=2.35, p=.02$ ).

With regard to the exact number of candies that children shared, we found that fully $44 \%$ of 3 - and 4-year-olds gave up two Smarties, thereby exactly equalizing the outcome. One third of children shared one candy, whereas a further $22 \%$ shared three of the five candies.

\section{Cranking duration}

Cranking times are illustrated in Fig. 3. Inspection of this figure shows that children were faster cranking for high offers (i.e., 5-5 and 5-1) than for low offers (i.e., 1-1 and 1-5) and that older children needed less time than younger children. The final model of the GLMM supported this observation given that both value (estimate $=.46, t=8.93, p<.001$ ) and age group (estimate $=-.39, t=-4.34$, $p<.001$ ) were significant predictors of the cranking duration. We also found a main effect of equality (estimate $=.11, t=2.44, p=.02)$; that is, children were faster at accepting equal allocations $(M=11.33$ 


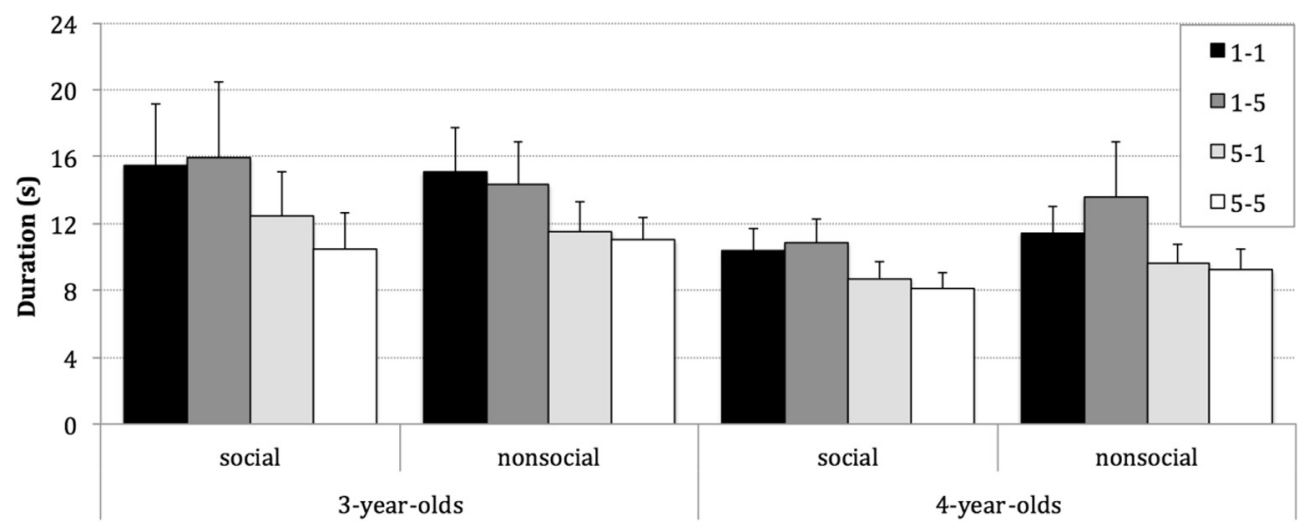

Fig. 3. Mean cranking durations for each allocation in Study 1. Error bars represent standard errors.

$\mathrm{s}, S D=4.71)$ than at accepting unequal allocations $(M=12.10 \mathrm{~s}, S D=5.52)$. Children got faster over the course of trials as well (main effect of trial number, estimate $=-.09, t=-3.71, p<.001$ ).

\section{Language}

Nearly all children (95\%) were able to label the contents of the cups correctly. In addition, $29 \%$ of 3 -year-olds and $42 \%$ of 4 -year-olds also spontaneously compared the bait in their cup with reference to the other cup. As illustrated in Fig. 4 and 3-year-olds often produced comparative language when they were facing equality but almost never if the allocation was unequal. Furthermore, these statements occurred more in the social condition. The 4-year-olds also differentiated between equal and unequal allocations and conditions in their comparative statements, although to a lesser extent than the 3-year-olds. Accordingly, analysis revealed significant interactions between age group and equality, $\operatorname{LRT}(1)=30.50, p<.001$, as well as between age group and condition, $L R T(1)=3.81, p=.05$.

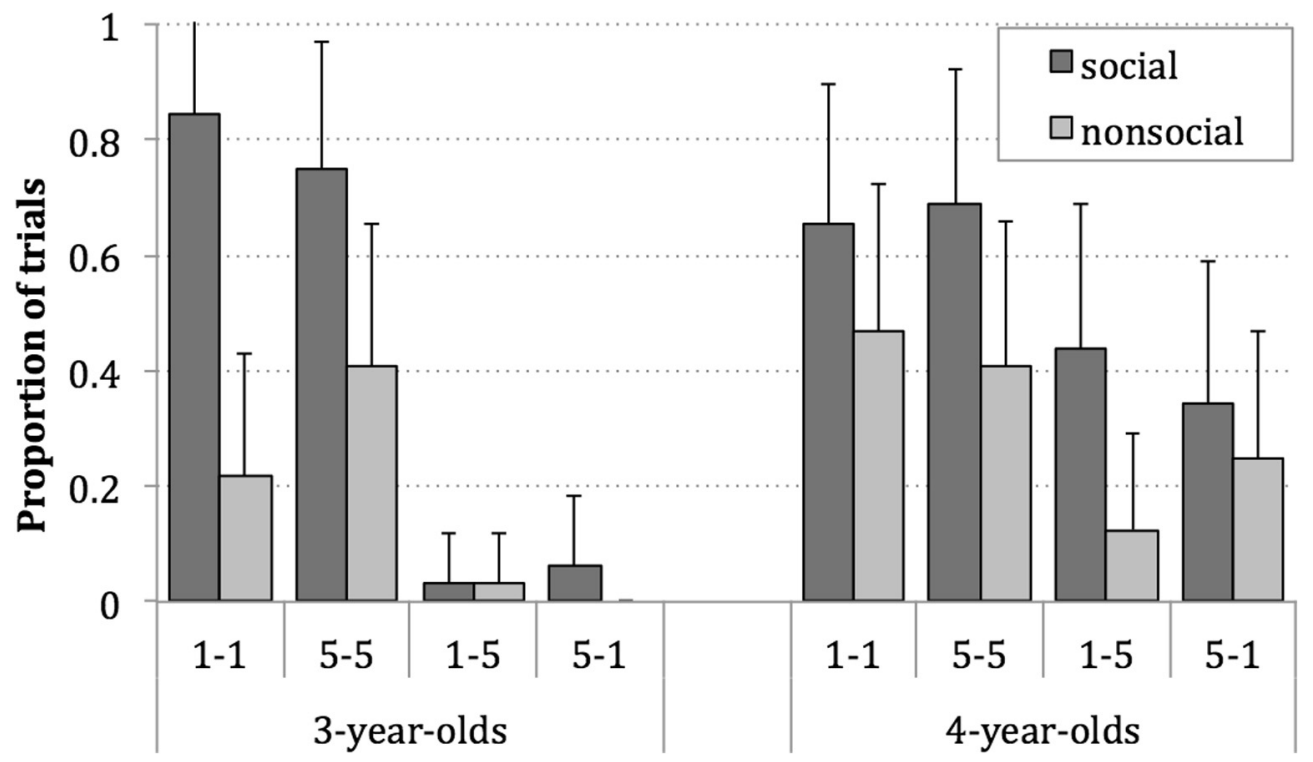

Fig. 4. Comparative statements for each allocation in Study 1. Error bars represent standard errors. 


\section{Discussion}

Study 1 found that 3- and 4-year-old children already show inequity aversion toward both advantageous and disadvantageous outcomes in situations where they interact with a social collaborative partner but not if they are on their own. Specifically, if children got less than the other, they showed their disapproval by claiming a share from their social partner or by rejecting the whole payoff. Crucially, if they received more than the other, they attempted to equalize the outcome either by sharing some of their own spoils or, in some cases, by rejecting all rewards. Equal outcomes, irrespective of whether they provided a high or low value, were mostly accepted. This is, to our knowledge, the first study to systematically investigate inequity aversion toward both advantageous and disadvantageous outcomes in a within-participants design and with children this young.

Our findings are congruent with Blake and McAuliffe (2011) in showing that children dislike receiving less than others (disadvantageous inequity) from early on. But we found that disadvantageous inequity aversion is already present in 3-years-olds in our experimental paradigm-that is, when they received less than their partner, children either rejected the distribution or requested candies from their partner-and, moreover, only in the social condition, implying a social comparison. Therefore, we did not replicate previous findings claiming that there is aversion to unequal distributions also in nonsocial situations (McAuliffe et al., 2013). Given the age differences in the studies (and a different role for the adult), this could indicate a developmental difference. During their early years (the focus of the current study), children might need a social partner who receives more than they do to object and react negatively toward an unequal distribution of rewards. Later on in development and in situations with an adult distributor (the focus of McAuliffe et al., 2013), children might be objecting to unfair adult behavior even without a peer partner present.

In our study, we provided rewards that belonged to nobody in the first place and that were distributed in an equal or unequal manner by accident. Although there is evidence that it should not influence children's subsequent behavior whether allocations were generated deliberately or randomly, at least in a social context (McAuliffe et al., 2013), it might be that the way in which rewards are allocated makes a difference in a nonsocial situation. In McAuliffe and colleagues' (2013) second experiment, comparing a social scenario against a nonsocial scenario, it was the experimenter who distributed candies, whereas in our study this happened-from children's perspective-by accident. This might be part of the reason why in our study children rarely reacted aversively toward inequality in the nonsocial context, whereas McAuliffe and colleagues found that children still rejected disadvantageous allocations when no social partner was present. In that case, children might have been rejecting the offer as a way to punish the experimenter for making the unfavorable allocation.

Most surprising, we also found that 3- and 4-year-old children dislike receiving more than others, thereby showing aversion to advantageous inequity even if this required a sacrifice (giving candies) on their part. This is with children up to 5 years younger than reported so far (see Blake \& McAuliffe, 2011; McAuliffe et al., 2013). The fact that it occurred only when a social collaborative partner was present but not in a nonsocial situation supports the similar results of Hamann and colleagues (2011) and suggests that the process really is one of social comparison. This result differs from that of previous studies (e.g., Blake \& McAuliffe, 2011; McAuliffe et al., 2013) mainly because we used a more inclusive set of measures. In particular, we looked not just at children's rejections of the proposed distribution-a very demanding measure in that it punishes both participants-and also included measures of children's attempts to equalize rewards. Indeed, children rejected only rarely in our study, perhaps because it is especially hard for very young children to reject an offer of candies right in front of them due to a lack of impulse control (e.g., Steinbeis et al., 2012) but also because our crank mechanism gave children time to think about their decision and even provided them with the opportunity to change their decision during the trial. In fact, $19 \%$ of 3 -year-olds and $6 \%$ of 4 -year-olds corrected their decisions; that is, they started rejecting the allocations but then changed the cranking direction to accept the offer in at least one trial. In addition, in the current study, rejected candies genuinely disappeared and were not taken away by the experimenter, such that the child could have still hoped to get them later. This modification of the procedure, together with the fact that the social situation had two agents not only facing one another but actually collaborating, might also explain why 
in the current study inequity aversion was confined to the social condition, whereas there were almost no aversive responses in the nonsocial situation.

With regard to the exact number of candies that children shared, we defined this behavior as attempts to correct the unequal payoff that includes, but is not restricted to, strict equalizations. Therefore, it is not crucial whether children shared one candy or two candies (or even more) because either number shows their willingness to make an unequal payoff more fair. Nevertheless, the majority of children shared two candies, which restored an equal resource allocation. Overall, all children gave up within the range of one to three resources, but crucially no child gave up more candies than that. Given the young age of participants, one can argue that it would have been difficult for them to numerically even out the outcome without additional help with counting. The key finding, thus, is that they acted toward an equal outcome even if they did not exactly equalize the payoff all the time.

The preference of equal outcomes was observed not only in children's aversive reactions, such as sharing, requesting, and rejecting, but also in their verbal statements. Children especially acknowledged equal distributions, whereas they commented less when the offer was unequal (in fact, 3-year-olds in this case rarely commented at all) and also differentiated between the social and nonsocial situations, producing more statements generally when they were interacting with a collaborative partner.

Furthermore, inequity aversion was also evident in the time measures. Here, we did not look at how long children needed to decide what to do; rather, we looked at how long it took them to actually carry out their decision as a possible measure of the underlying motivation to obtain their rewards. In line with the main results, children took longer (i.e., cranked more slowly) if they were to accept an unequal allocation. They were faster, and thus presumably more motivated, if the outcome was equal. In addition, the cranking time measures provide us with another major insight, namely that children were generally faster when the allocation promised a high outcome for them (5-5 or 5-1). Against this background, the evidence of advantageous inequity aversion in young children appears to be even more astonishing; despite the fact that children were particularly keen to receive a high number of rewards, many were still willing to share their rewards (or even reject them) in order to equalize with their partner.

In sum, findings from Study 1 indicate that children show inequity aversion from as early as 3 years of age. Children showed a variety of aversive reactions (sharing, requesting, and rejecting) in order to prevent a partner from receiving a more desirable allocation (disadvantageous inequity) or even a less desirable allocation (advantageous inequity). Notably, all possible allocations were tested within participants and also with regard to a possible confound with value. The analysis of children's verbal statements made clear that children were highly aware of the number of Smarties in the cups and differentiated between high and low values accordingly. Time measures then indicated that value played a role in how motivated children were to receive the outcome, but this bias for high payoffs was then overwritten by the preference for equality. Moreover, both types of inequity aversion were closely tied to situations in which children were paired with a social collaborative partner, suggesting that this inequity aversion is a genuinely social phenomenon and cannot be simply explained by a preference for symmetry. This does not rule out the possibility that, later in development, a general aversion toward inequity transfers beyond its social context to nonsocial settings as well, especially in the case of disadvantageous inequity aversion.

In all, the early emergence of both types of inequity aversion allows us to assume that they both originate from a general sense of fairness, ${ }^{2}$ although the particular psychological mechanisms behind them (e.g., envy and spite for disadvantageous allocations, reputational concerns and generosity for advantageous allocations) might be different and might follow different developmental trajectories. Further research is needed in order to shed light on the underpinning developmental mechanisms in detail. For a start, the strong social component of both types of inequity aversion supports an evolutionary explanation about how and why inequity aversion could have evolved given that it requires, to some extent, personal sacrifices. The current findings fit well to the theoretical assumptions known as social

\footnotetext{
${ }^{2}$ A follow-up study provides some evidence that both types of inequity aversion might occur already at 2 years of age, albeit only for girls; see Study 3 reported in the online supplementary material.
} 
hypothesis, according to which inequity aversion evolved to regulate cooperative interactions as it prevents individuals from being exploited by others or from exploiting others (Brosnan, 2011; Fehr \& Schmidt, 1999).

\section{Study 2}

The question now is whether inequity aversion is present in humans' nearest primate relatives, who do not have the same kind of evolutionary history of cooperation and obligate mutualism as humans (Hill, 2002; Sterelny, 2007; Tomasello, 2009). Study 2, therefore, tested whether humans' closest living relative, the chimpanzee (Pan troglodytes), would show a similar pattern of inequity aversion as human children in a similar study design or, as predicted, would not differ in their reactions to distributions in social and nonsocial (no partner and so no social inequity) conditions.

\section{Method}

\section{Subjects}

Subjects were nine socially housed chimpanzees (for details, see Table 3 ) in the Wolfgang Köhler Primate Research Center at the Leipzig Zoo (Leipzig, Germany). Two additional chimpanzees were excluded as subjects because they failed to demonstrate an understanding of the apparatus during the training sessions. Depending on condition, subjects were tested both individually and separately with two different partners. Two high-ranking male members of the same social group were selected as partners. All tested chimpanzees had taken part in a variety of tests of physical and social cognition in the past, some including a cooperative task involving rope pulling (Greenberg, Hamann, Warneken, \& Tomasello, 2010; Hamann et al., 2011), but the specific testing paradigm for this study was novel to the subjects. Testing was conducted in their sleeping rooms by a familiar experimenter (E). Subjects had ad libitum access to water and were not food deprived.

\section{Design}

All subjects were tested in two conditions (social and nonsocial) in a within-subjects design. Each subject participated in four test sessions consisting of two nonsocial sessions and two social sessions featuring two different partners. We counterbalanced order of condition, with half of the chimpanzees starting with the social condition and the other half starting with the nonsocial condition. Each session consisted of eight trials presenting four different reward allocations as described in Study 1 (see Table 1).

\section{Materials}

The overall functionality of the apparatus was closely modeled to the children's version of Study 1. The apparatus was mounted in an experimental booth in the center of the testing room and consisted of three mesh panels and one open side ( $1.2 \mathrm{~m}$ wide $\times 1.0 \mathrm{~m}$ long). When tested in the social condition, two chimpanzees could sit on either side of the booth facing mesh panels. When tested in the nonsocial condition, the left mesh panel was replaced by a solid panel, restricting access to the booth. The experimenter operated the apparatus from a central position at the open side of the booth.

The apparatus consisted of two levels (for details, see Fig. 5). The upper level consisted of a sliding platform holding rewards outside chimpanzees' reach. To deliver rewards to the lower level of the apparatus, the platform needed to be moved, either by two chimpanzees simultaneously pulling two ends of a rope that were attached to the board (adapted from Hirata \& Fuwa, 2007, and Melis, Hare, \& Tomasello, 2006) or, alternatively, by the experimenter pulling the rope alone. The rewards were then collected by two small centrally located cups on the second level. The cups were mounted on two sliding platforms and could be moved along a track. Chimpanzees sitting on the right side of the booth had access to a strap that was attached to the sliding platforms. By pulling the strap, the two platforms with attached plates moved outward and were eventually accessible through the meshes on the left and right sides of both. There was a slider installed between both levels of the apparatus with which the experimenter could manipulate the allocation of rewards between the two cups. With the 
Table 3

Chimpanzee subjects and their kin relationships with partners.

\begin{tabular}{llllll}
\hline Subject & Sex & Age (years) & Rearing & Kin relation \\
\cline { 4 - 5 } & & & Robert & Lome \\
\hline Bangolo & Male & 5 & Biological mother & Grandfather & Uncle \\
Corrie & Female & 38 & Hand reared & None & Mother \\
Dorien & Female & 34 & Hand reared & None & None \\
Kara & Female & 9 & Biological mother & Daughter & Half-sister \\
Natascha & Female & 35 & Hand reared & None & None \\
Riet & Female & 37 & Hand reared & None & None \\
Sandra & Female & 21 & Biological mother & Daughter & Half-sister \\
Tai & Female & 12 & Biological mother & Daughter & Half-sister \\
Ulla & Female & 37 & Hand reared & None & None \\
\hline
\end{tabular}

exception of one subject who received pellets during the training sessions, grapes were used as rewards for training purposes. All testing was conducted with cherry tomatoes.

\section{Procedure}

Training sessions. The purpose of the training sessions was to ensure that the chimpanzees understood how the apparatus in the two different test conditions worked.

1. Reward allocation. Training began with a familiarization of chimpanzees with the two plates on the sliding platforms. The experimenter placed rewards randomly in either a 5-1 or 1-5 allocation on the two centrally located plates and shoved them by hand to the left and right sides of the booth. Chimpanzees were placed opposite the experimenter in a central position. Once the plates were moved, chimpanzees could obtain the rewards through the mesh. Crucially, chimpanzees could reach only one plate at one side at a time. The experimenter then removed the rewards that were left on the opposite side. The purpose of these first training trials was to determine whether chimpanzees correctly understood that the plates were baited with a different number of rewards and to measure their preference for a higher payoff independent of location. When subjects succeeded (i.e., by correctly choosing the side with five rewards first) on three consecutive trials, subjects advanced to the next training phase. All but one subject passed this training phase quickly within the first three or four trials. The one subject having difficulty passed after pellets were introduced as rewards.

2. Familiarization strap. During this phase, chimpanzees demonstrated that they knew to pull the end of the strap to gain access to the rewards on the two plates. Therefore, subjects were placed on the right side of both. The plates were baited with two grapes each. Chimpanzees were then given the strap ready to pull. Nearly all subjects successfully pulled the strap and, thus, moved the containers into reach. They then showed that they knew that rewards were accessible on both sides because they first reached through the mesh on the right side to retrieve their rewards and then went over to the left side to obtain the remaining rewards. One subject could not be encouraged to pull the strap in a first trial but mastered the task in the second trial.

3. Mesh panel control. The purpose of this control was to determine whether chimpanzees understood, for an open mesh setup, that rewards were accessible on either side of the booth, which is why pulling the strap would be a rewarding strategy even if the plate on their own (right) side contained no rewards. Therefore, chimpanzees were expected to pull the strap and retrieve rewards when rewards were allocated in both a 0-2 and 2-0 manner. Each allocation was presented in blocks of two trials. Chimpanzees proceeded to the test phase if they succeeded in three subsequent trials of each allocation. Seven subjects passed this phase on the first try, whereas two subjects passed after a second session.

4. Solid panel control. During this phase, subjects demonstrated that they understood that, when the mesh panel on the left was replaced with a solid panel, rewards on the left could not be accessed anymore. Therefore, subjects were expected to reject pulling the strap (operationalized by not pulling the containers into reach within $60 \mathrm{~s}$ ) if the left plate contained two rewards but the right plate contained no bait. This allocation was presented in blocks of two trials intermitted by reverse allocations 

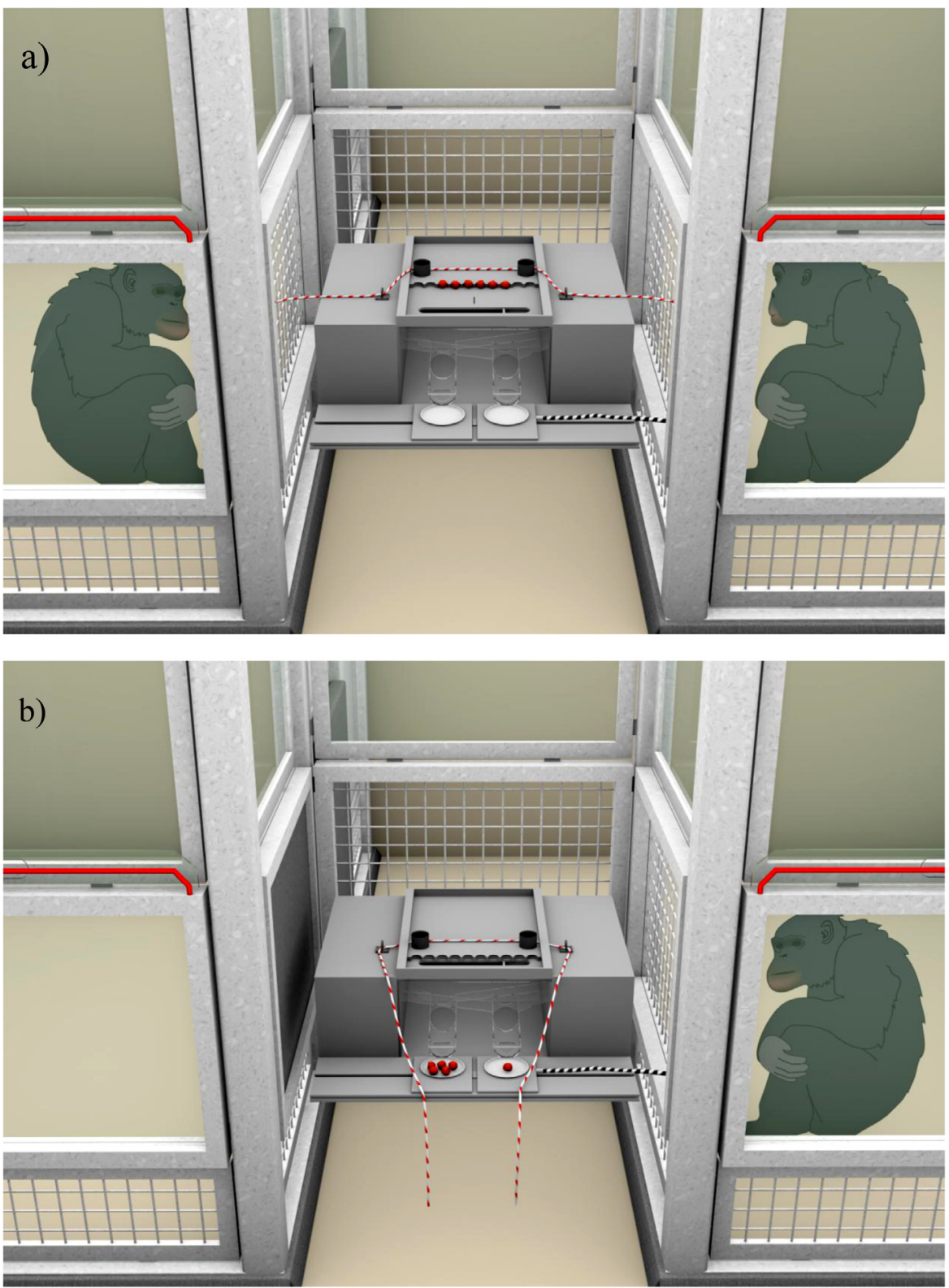

Fig. 5. Illustration of the test apparatus in the social (A) and nonsocial (B) conditions in Study 2. Subject chimpanzees were placed on the right side of the booth, while the partner (if present) sat on the left side of the booth. Rewards were placed in the center of the flat and could not be reached. A little platform was used to shove the rewards forward and cause them to fall down into two cups. In the social condition, the platform could be moved only by means of a rope that was threaded through metal loops attached to the platform. When both chimpanzees took both ends of the rope and pulled simultaneously, the platform would shove the rewards forward. If only one chimpanzee pulled one end, the rope would unthread, leaving the board stationary and rewards inaccessible. Given the dimensions of the booth, one chimpanzee was not able to touch and hence pull both ends of the rope alone. In the nonsocial condition, the experimenter operated the platform by pulling it in his direction by means of a single rope. With the help of a slider, the final split-up, and thus the final bait of the plates, could be manipulated. Only subjects on the right side had access to a strap. Pulling the strap caused the plates to move outward, where chimpanzees could obtain the rewards through the mesh panel ("accepting an allocation"). 
(where pulling the rope would make sense) as distraction trials. Again, chimpanzees needed to be successful, and thus reject pulling the rope, in three consecutive trials. Because this task was naturally more demanding than the other training phases (e.g., because it took subjects a few trials to realize that the left panel had been replaced with a solid one), only three chimpanzees passed this phase on the first attempt. The remaining subjects proceeded to the next training phase after an average of two more sessions.

5. Familiarization of full apparatus. This training phase familiarized subjects with the two-step functioning of the full apparatus, including pulling the rope on the upper level and then pulling the strap to retrieve the rewards on the lower level.

For the social condition, subjects were placed on the right side of the booth and partners were placed on the left side. The experimenter baited the upper level with four rewards visible for both chimpanzees. The experimenter then strung each end of the rope that was attached to the platform through the mesh on both sides of the booth. Because most subjects $(n=5)$ and the two partner chimpanzees had participated in a similar training phase as part of previous studies (e.g., Greenberg et al., 2010; Hamann et al., 2011), most pairs passed this first part immediately; they simultaneously pulled the ends of the rope, which caused the rewards to fall down onto the two plates (two rewards per plate) on the lower level of the apparatus. The experimenter then released the strap for subjects. A trial was considered as successful if the subject pulled the strap within $60 \mathrm{~s}$ and both chimpanzees obtained their rewards.

For the nonsocial condition, subjects were tested individually without a partner. A solid panel closed the left side access toward the rewards. The trial started again with the experimenter baiting the platform on the upper level with four rewards. The experimenter then pulled the rewards forward himself. After rewards had fallen onto the lower level in a 2-2 split between plates, the experimenter released the strap for subjects. A trial was considered as successful if the subject pulled the strap within $60 \mathrm{~s}$ and obtained rewards on his or her side.

Each test session began with two trials of the familiarization training matching the condition in which subjects were tested. All but two subjects passed the two trials immediately and proceeded with the test trials in all test sessions. Two subjects (and their partners) in the social condition did not successfully manage the simultaneous rope pulling in a first attempt and needed a second and third training session, respectively, before they could eventually proceed with the test trials.

Test sessions. Each test session began with two refreshment trials of the last training phase matching the condition in which subjects were tested. Each test session applied four different allocations of rewards. Each trial began with the experimenter holding up new rewards and placing them in the center on the board on the upper level of the apparatus. In a next step, rewards were then moved onto the lower level of the apparatus either by the subject and the partner simultaneously pulling a rope together (social condition) or by the experimenter alone (nonsocial condition). After all rewards had fallen into the plates in the correct split-up, the experimenter released the strap for the subject. A trial ended once the subject had completely pulled the rope and after all rewards had been eaten or until $60 \mathrm{~s}$ had elapsed. In the latter case, the experimenter removed the rewards and started a new trial. In cases where rewards were allocated incorrectly due to apparatus malfunction ( $<5 \%$ of trials), the experimenter did not release the strap but removed the rewards from the containers and repeated the trial.

\section{Coding and data analysis}

Coding was done from video by the first author. Primary coding focused on chimpanzees' aversive behavior, which consisted of the following two behavioral categories: rejecting (i.e., not pulling the strap within a 60-s time frame; changing sides with the partner) and requesting behavior (including begging and stealing rewards from the partner and walking over to the opposite side touching the panel). Unsuccessful stealing or begging attempts were also coded as requesting behavior. Because coding focused exclusively on the subject chimpanzee, instances of forced sharing (i.e., when the partner walked over and stole rewards from the subject) were not coded as aversive behavior.

We also measured how long chimpanzees took to pull the strap in order to move the rewards within reach. Duration was coded from the moment the experimenter released the strap until subjects 


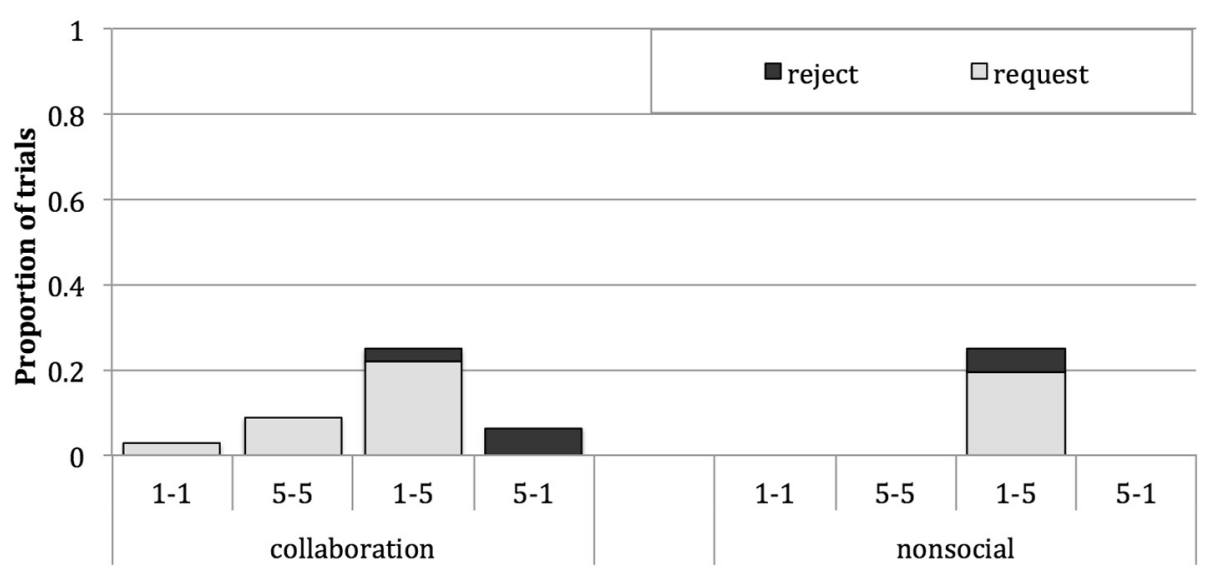

Fig. 6. Proportions of trials in Study 2 where chimpanzees showed aversive behavior toward four different resource allocations $(1-1,5-5,1-5$, and 5-1).

first touched their container. One quarter (25\%) of trials in each condition were randomly selected and coded by a second observer blind to the hypothesis of the study. Inter-rater agreement was excellent for both aversive behavior $(\kappa=.93)$ and pulling duration $(r=.96)$. Statistical analysis followed the methods as described in Study 1, with the full model including condition (social or nonsocial), reward allocation $(1-1,5-5,1-5$, or $5-1)$, trial number $(1,2,3,4,5,6,7$, or 8$)$, and test session. Pre-analysis revealed that there was no effect of rearing or kin relation; thus, these factors were excluded from the final analysis. There was also no performance difference with regard to the two partners.

Results

The final model revealed no effect of trial number $(p=.33$ ), session $(p=.08)$, or condition (social or nonsocial; $p=.78$ ) on chimpanzees' aversive behavior. However, there was a significant main effect of resource allocation, $\operatorname{LRT}(3)=15.06, p=.002$. Inspection of Fig. 6 and the final model estimates reveals that this main effect was solely due to the disadvantageous allocation (1-5), which differed significantly from the three alternative allocations $(z s<3.37, p s \leqslant .001)$.

There were five instances in which the partner in the social condition enforced a share if the partner was at a relative disadvantage; four of these cases involved the two youngest subjects of the sample. Regarding how long chimpanzees took to pull the strap, the comparison between the full model, containing all possible predictors, and its respective null model, including only subject identity, showed no significant result $(p=.15)$. Hence, none of the predictors had a significant influence on pulling duration.

\section{Discussion}

The second study revealed that chimpanzees disliked disadvantageous outcomes; aversive behavior (mostly in the form of requesting behavior) occurred only if the number of food items offered on subjects' side was less than what was offered on the opposite side, but not if subjects' side was favored. Importantly, subjects' reactions were not affected by the presence of a social partner. Thus, what guided chimpanzees' reaction in these cases was the (unfavorable) numerical discrepancy between the food on the two plates-irrespective of whether the food on the opposite plate was available for a conspecific or for nobody. The fact that there was no influence of the social context, as well as no evidence of advantageous inequity aversion, speaks for the fact that primates did not take the partner's outcome into consideration. It can rather be assumed that their reactions were primarily 
based on personal gain (Jensen et al., 2006). However, if maximizing their gain was the only incentive driving their aversive behavior, we would have expected to see similar behavior in all allocation scenarios where the opposite plate offered five food items. The fact that chimpanzees mainly objected to unfavorable unequal food offers (in the form of increased begging and requesting) suggests that their main concern was maximizing their own resources.

The most likely explanation for their negative reactions is that chimpanzees were simply expecting to get the same (greater) amount of food that was offered on the opposite site (food expectation hypothesis; see Bräuer et al., 2006). Thus, these findings support previous findings indicating that chimpanzees are indifferent to the social nature of a food acquisition context and, as such, are not other-regarding (e.g., Jensen et al., 2006; Silk et al., 2005). Given the overall consistent finding in the literature that nonhuman primates are generally not very good at solving fairness problems (e.g., Jensen et al., 2006, 2007; Kaiser et al., 2012; Silk et al., 2005), it can be assumed that their aversion toward unequal disadvantageous distributions is not genuinely motivated by fairness concerns. Interestingly and importantly, this is not because they have no prosocial motivations at all. Chimpanzees help others to attain their goals in a variety of situations (see Warneken \& Tomasello, 2009, for a review), including to obtain food if they themselves have no chance of getting any (Melis, Schneider, \& Tomasello, 2011). Thus, one hypothesis is that chimpanzees and other great apes have a basic sympathy for others in need, but a sense of fairness requires an impartial perspective in which the needs of all individuals are equally balanced, and this seems to be beyond their capacities (Tomasello, 2016).

\section{Conclusion}

Combined, the current two studies provide the most detailed picture to date of young children's and chimpanzees' responses toward unequal payoffs. The strong social component of inequity aversion in humans is assumed to originate from an evolutionary mechanism regulating successful cooperative interactions (e.g., Baumard, André, \& Sperber, 2013; Fehr \& Schmidt, 1999; Tomasello, 2009). The important role of inequity aversion also characterizes modern society, of course, ranging from promoting equal pay for men and woman to making charitable contributions to the poor.

In contrast, for humans' closest living relative, chimpanzees, the mechanism is not a sense of fairness but rather a concern with maximizing resources for the self. This orientation-and despite the presence of some forms of prosocial motivation in chimpanzees-is explained by the fact that nonhuman primates lack an evolutionary history of mutualistic cooperation (Sterelny, 2007; Tomasello, 2009, 2016), and so inequity aversion-in the form that it occurs in humans from early in ontogeny-has never played an important role in their evolution and, therefore, is not part of their current behavioral repertoire.

In all, the current results underscore the importance for humans, even very early in ontogeny, for a motivation to treat others fairly and an expectation to be treated fairly by others. Only through such behavior can one expect to be viewed positively as a cooperative member of the social group and so reap all of the rewards that come with such membership. The degree to which these motivations and expectations depend on adult socialization practices awaits further research, perhaps especially crosscultural research in which children who are taught differently about fairness are compared in this or a similar paradigm. It is even imaginable that, rather than cultural learning, ecological factors such as the value of fairness for securing cooperative partners across different situations might be the decisive force (e.g., Debove, André, \& Baumard, 2015; Delton, Krasnow, Cosmides, \& Tooby, 2010).

\section{Acknowledgments}

We thank all the children and their families involved in this work for their cooperation, and we thank E. Rossi and J. Grossmann for their coordination and great assistance throughout the realization of this project as well as S. Bölke, J. Bräuer, M. Müller-Heuss, A. Tomm, and H. Stühring for their help in data collection and behavior coding. We also thank R. Pieszek for building the apparatuses, P. Jahn for providing technical support, S. Tüpke for creating the figures, R. Mundry for giving statistical advice, 
and the zookeepers at Leipzig Zoo for assisting with the chimpanzees. This work was supported by a German National Academic Foundation stipend for J. Ulber.

\section{Appendix A. Supplementary data}

Supplementary data associated with this article can be found, in the online version, at http://dx.doi. org/10.1016/j.jecp.2016.10.013.

\section{References}

Baayen, R. H., Davidson, D. J., \& Bates, D. M. (2008). Mixed-effects modeling with crossed random effects for subjects and items. Journal of Memory and Language, 59, 390-412.

Bates, D., Maechler, M., Bolker, B., \& Walker, S. (2014). Ime4: Linear mixed-effects models using Eigen and S4. R package version 1.1-7. Available at: http://CRAN.R-project.org/package=lme4.

Baumard, N., André, J., \& Sperber, D. (2013). A mutualistic approach to morality: The evolution of fairness by partner choice. Behavioral and Brain Sciences, 36, 59-78.

Birch, L. L., \& Billman, J. (1986). Preeschool children's food sharing with friends and acquaintances. Child Development, 57 , 387-395.

Blake, P. R., \& McAuliffe, K. (2011). "I had so much it didn’t seem fair”: Eight-year-olds reject two forms of inequity. Cognition, 120, 215-224.

Blake, P., McAuliffe, K., Corbit, J., Callaghan, T., Barry, O., ... Bowie, A. (2015). The ontogeny of fairness in seven societies. Nature, $528,258-261$.

Blake, P., McAuliffe, K., \& Warneken, F. (2014). The developmental origins of fairness: The knowledge-behavior gap. Trends in Cognitive Sciences, 18, 559-561.

Boesch, C. (1994). Cooperative hunting in wild chimpanzees. Animal Behavior, 48, 653-667.

Bräuer, J., Call, J., \& Tomasello, M. (2006). Are apes really inequity averse? Proceedings of the Royal Society B: Biological Sciences, $273,3123-3128$.

Bräuer, J., Call, J., \& Tomasello, M. (2009). Are apes inequity averse? New data on the token-exchange paradigm. American Journal of Primatology, 71, 175-181.

Brosnan, S. F. (2011). A hypothesis of the co-evolution of cooperation and responses to inequity. Frontiers in Neuroscience, 5. http://dx.doi.org/10.3389/fnins.2011.00043.

Brosnan, S. F., \& de Waal, F. (2003). Monkeys reject unequal pay. Nature, 425, 297-299.

Brosnan, S., Schiff, H., \& de Waal, F. (2005). Tolerance for inequity may increase with social closeness in chimpanzees. Proceedings of the Royal Society B: Biological Sciences, 272, 253-258.

Brosnan, S. F., Talbot, C., Ahlgren, M., Lambeth, S. P., \& Schapiro, S. J. (2010). Mechanisms underlying responses to inequitable outcomes in chimpanzees, Pan troglodytes. Animal Behaviour, 79, 1229-1237.

Dawes, C. T., Fowler, J. H., Johnson, T., McElreath, R., \& Smirnov, O. (2007). Egalitarian motives in humans. Nature, 446, 794-796.

Debove, S., André, J.-B., \& Baumard, N. (2015). Evolution of equal division among unequal partners. Evolution, 69, $561-569$.

Delton, A. W., Krasnow, M. M., Cosmides, L., \& Tooby, J. (2010). Evolution of fairness: Rereading the data. Science, $329,389$.

Dubreuil, D., Gentile, M. S., \& Visalberghi, E. (2006). Are capuchin monkeys (Cebus apella) inequity averse? Proceedings of the Royal Society B: Biological Sciences, 273, 1223-1228.

Fehr, E., \& Schmidt, K. (1999). A theory of fairness, competition, and cooperation. Quarterly Journal of Economics, 114, 817-868.

Fontenot, M., Watson, S., Roberts, K., \& Miller, R. (2007). Effects of food preferences on token exchange and behavioural responses to inequality in tufted capuchin monkeys, Cebus apella. Animal Behaviour, 74, 487-496.

Frydman, O., \& Bryant, P. (1988). Sharing and the understanding of number equivalence by young children. Cognitive Development, 3, 323-339.

Gilby, I. C. (2006). Meat sharing among the Gombe chimpanzees: Harassment and reciprocal exchange. Animal Behavior, 71 , 953-963.

Gräfenhain, M., Carpenter, M., \& Tomasello, M. (2013). Three-year-olds' understanding of the consequences of joint commitments. PLOS ONE, 8(9), e73039.

Greenberg, J., Hamann, K., Warneken, F., \& Tomasello, M. (2010). Chimpanzee helping in collaborative and noncollaborative contexts. Animal Behaviour, 80, 873-880.

Hamann, K., Warneken, F., Greenberg, J. R., \& Tomasello, M. (2011). Collaboration encourages equal sharing in children but not in chimpanzees. Nature, 476, 328-331.

Hill, K. (2002). Altruistic cooperation during foraging by the Ache, and the evolved human predisposition to cooperate. Human Nature, 13, 105-128.

Hirata, S., \& Fuwa, K. (2007). Chimpanzees (Pan troglodytes) learn to act with other individuals in a cooperative task. Primates, 48, $13-21$.

Hopper, L., Lambeth, S., Schapiro, S., \& Brosnan, S. (2013). When given the opportunity, chimpanzees maximize personal gain rather than "level the playing field". PeerJ, 1. http://dx.doi.org/10.7717/peerj.165.

Jensen, K., Call, J., \& Tomasello, M. (2007). Chimpanzees are rational maximizers in an ultimatum game. Science, 318, 107-109.

Jensen, K., Hare, B., Call, J., \& Tomasello, M. (2006). What's in it for me? Self-regard precludes altruism and spite in chimpanzees. Proceedings of the Royal Society B: Biological Sciences, 273, 1013-1021.

Kaiser, I., Jensen, K., Call, J., \& Tomasello, M. (2012). Theft in an ultimatum game: Chimpanzees and bonobos are insensitive to unfairness. Biology Letters, 8, 942-945.

LoBue, V., Nishida, T., Chiong, C., DeLoache, J. S., \& Haidt, J. (2011). When getting something good is bad: Even three-year-olds react to inequality. Social Development, 20, 154-170. 
McAuliffe, K., Blake, P. R., Kim, G., Wrangham, R. W., \& Warneken, F. (2013). Social influences on inequity aversion in children. PLOS ONE, 8(12), e80966.

McAuliffe, K., Chang, L. W., Leimgruber, K. L., Spaulding, R., Blake, P. R., \& Santos, L. R. (2015). Capuchin monkeys, Cebus apella, show no evidence for inequity aversion in a costly choice task. Animal Behaviour, 103, 65-74.

Melis, A. P., Hare, B., \& Tomasello, M. (2006). Engineering cooperation in chimpanzees: Tolerance constraints on cooperation. Animal Behaviour, 72, 275-286.

Melis, A., Schneider, A., \& Tomasello, M. (2011). Chimpanzees, Pan troglodytes, share food in the same way after collaborative and individual food acquisition. Animal Behaviour, 82, 485-493.

Mitani, J. C., \& Watts, D. (2001). Why do chimpanzees hunt and share meat? Animal Behavior, 61, 915-924.

Olson, K. R., \& Spelke, E. S. (2008). Foundations of cooperation in young children. Cognition, 108, 222-231.

Peterson, C., Peterson, J., \& McDonald, N. (1975). Factors affecting reward allocation by preschool children. Child Development, 46, 942-947.

Rakoczy, H., Warneken, F., \& Tomasello, M. (2009). Young children's selective learning of rule games from reliable and unreliable models. Cognitive Development, 24, 61-69.

Rochat, P., Dias, M. D. G., Guo, L. P., Broesch, T., Passos-Ferreira, C., Winning, A., \& Berg, B. (2009). Fairness in distributive justice by 3- and 5-year-olds across seven cultures. Journal of Cross-Cultural Psychology, 40, 416-442.

Roma, P. G., Silberberg, A., Ruggiero, A. M., \& Suomi, S. J. (2006). Capuchin monkeys, inequity aversion, and the frustration effect. Journal of Comparative Psychology, 120, 67-73.

Shaw, A., \& Olson, K. R. (2012). Children discard a resource to avoid inequity. Journal of Experimental Psychology: General, 141, 382-395.

Sheskin, M., Ashayeri, K., Skerry, A., \& Santos, L. R. (2014). Capuchin monkeys (Cebus apella) fail to show inequality aversion in a no-cost situation. Evolution and Human Behavior, 35, 80-88.

Sheskin, M., Bloom, P., \& Wynn, K. (2014). Anti-equality: Social comparison in young children. Cognition, 130, 152-156.

Silk, J. B., Brosnan, S. F., Vonk, J., Henrich, J., Povinelli, D. J., Richardson, A. S., ... Schapiro, S. J. (2005). Chimpanzees are indifferent to the welfare of unrelated group members. Nature, 427, 1357-1359.

Steinbeis, N., Bernhardt, B. C., \& Singer, T. (2012). Impulse control and underlying functions of the left DLPFC mediate agerelated and age-independent individual differences in strategic social behavior. Neuron, 73, 1040-1051.

Sterelny, K. (2007). Social intelligence, human intelligence, and niche construction. Philosophical Transactions of the Royal Society B: Biological Sciences, 362, 719-730.

Tomasello, M. (2009). Why we cooperate. Cambridge, MA: MIT Press.

Tomasello, M. (2016). A natural history of human morality. Cambridge, MA: Harvard University Press.

Ulber, J., Hamann, K., \& Tomasello, M. (2015). How 18- and 24-month-old peers divide resources among themselves. Journal of Experimental Child Psychology, 140, 228-244.

Ulber, J., Hamann, K., \& Tomasello, M. (2016). Extrinsic rewards diminish costly sharing in 3-year-olds. Child Development, 87, 1192-1203.

Warneken, F., Lohse, K., Melis, A. P., \& Tomasello, M. (2011). Young children share the spoils after collaboration. Psychological Science, 22, 267-273.

Warneken, F., \& Tomasello, M. (2009). Varieties of altruism in children and chimpanzees. Trends in Cognitive Science, 13, 397-402. 